\title{
ENSEÑABILIDAD DE NORMAS INTERNACIONALES DE INFORMACIÓN FINANCIERA E INCIDENCIA EN LA DOCENCIA UNIVERSITARIA ${ }^{\prime}$
}

\author{
Yuleida Ariza Angarita ${ }^{2}$
}

Corporación Universidad de la Costa - Artículo Tipo 1. Investigación Científica y Tecnológica - Recibido: 19 de mayo 2014 - Aceptado: 24 de junio 2015

\section{RESUMEN}

En los escenarios actuales, se observa la necesidad de que los profesionales contables estén en capacidad de contribuir a la solución de problemas sociales complejos al mismo tiempo que participan en el progreso de las organizaciones, generando avances disciplinares que incorporen miradas inter y transdisciplinares con visión universal sin pérdida de identidad contextualizada. El presente artículo de investigación tiene como objetivo, analizar la práctica docente empleada en los procesos de enseñabilidad de las Normas Internacionales de Información Financiera, NIIF en la educación superior, de modo que responda con pertinencia a las competencias integrales que demanda la sociedad, orientado desde una metodología cuali-cuantitativa de tipo descriptivo y diseño no experimental. Dentro de los resultados, se menciona que las nuevas realidades mundiales demandan la necesidad de adaptar competencias internacionales en la formación del contador público. Se concluye la relevancia de un diseño de plan de asignaturas profundamente pensado que involucre elementos pedagógicos, axiológicos, culturales, cognitivos y metodológicos donde toma importancia una docencia alineada que incorpore pedagogías activas y proponentes para conducir aprendizajes significativos y pertinentes, todo un proceso de sensibilización reflexivo en el medio académico para mancomunadamente llegar a la superación disciplinar y promover la renovación del ejercicio docente.

\section{Palabras Clave:}

Normas Internacionales de Información Financiera, Práctica docente, enseñabilidad, Pedagogías activas.

JEL: A22, A23, M41.

Si va a referenciar este artículo

Ariza, Y. (2015). Enseñabilidad de normas internacionales de información financiera e incidencia en la práctica docente del contexto universitario, Económicas CUC, 36 (1), 57-70

\footnotetext{
${ }^{1}$ Artículo de Investigación científica, derivado de la investigación "Enseñabilidad de las normas internacionales de contabilidad y su incidencia en la práctica docente en la Universidad de la Costa, CUC", línea de estudios de contabilidad, Universidad de la Costa.

${ }^{2}$ Contador Público, Experto en Normas internacionales de Información financiera (NIIF), Especialista en Sistemas de Gestión de Calidad Integrados, Magister en Educación, Docente Investigador Universidad la Costa CUC, yariza9@cuc.edu.co
} 


\section{INTRODUCCIÓN}

Las interacciones entre enseñabilidad, aprendibilidad y educabilidad configuran un nuevo campo de investigación, dada su importancia para promover y alcanzar construcción de nuevos conocimientos, ello demanda un reto de teorización y una revisión crítica de los postulados que subyacen en tales conceptos con el propósito de comprender y resignificar la práctica pedagogía, especialmente de saberes de aplicación práctica aún difusa como es el tema de convergencia a Normas Internacionales de Información Financiera (NIIF) en Colombia; ello requiere la obtención de un conocimiento profundo, de sus bases de modo que provea de fundamento sus aplicaciones prácticas contextualizadas, lo cual hasta el momento se ha percibido carente de significado, conducido a través de metodologías dispersas con imprecisas interpretaciones y débiles resultados favorables al momento de ser adoptados.

Es así como, el evidente desequilibrio de los enfoques de formación en NIIF, algunas veces extremadamente academicistas y otras profesionista, desestiman la reflexión permanente de la realidad que invita a la superación disciplinar (Casinelli, 2008), esta desarticulación entre los procesos formativos de enseñabilidad, aprendibilidad y educabilidad, conllevan a reconocer que generar competencias profesionales no es solo poseer cantidad de conocimiento, sino que conducido de forma estimulante (enseñabilidad) posibilite la aproximación racional y crítica que el estudiante haga a la realidad (aprendibilidad) que parte de intereses propios y conocimientos previos para terminar en aprendizajes significativo y útil (educabilidad). Gallego \& Pérez (2011)
En este sentido Castillo \& Jesús (2010), establecen una aclaración sobre competencias cuando dicen:

Las competencias están condicionadas por factores ligústicos, sociales, históricos, culturales, etc., que las contextualizan. Por ello, las competencias deben integrar los saberes, y la aplicación práctica de esos saberes a la vida diaria de forma adecuada y en un contexto determinado. Los elementos que integran una competencia en general, son: saber, saber hacer, y saber ser. Además, hay que saber estar (p. 61).

En el mismo sentido, enseñar las NIIF sin establecer conexión con la realidad que permita su interpretación focalizada, puede desestimar el compromiso social de intervenir en las transformaciones sociales, de ello se establece la importancia de orientar el proceso formativo "hacia la formación de contadores para el mundo y no solamente para el desempeño local" (Avellaneda, 2010, p. 219), como vía para mejorar condiciones de vida, elemento fundamental de la función liberadora que produce la educación pertinente, analizada desde la mirada reflexiva de la realidad que trasciende en la intervención de la misma; pensamiento que es compartido por teóricos como Freire (1970), Habermas (2002) y Giroux (1990).

Autores como Carrasco (1996), Martínez (2001), Mattessich (2003), Gómez (2005); declararan la necesidad de una reorientación de la profesión contable, que demanda un conocimiento más universal, trascendente del positivismo normativo y pragmático que la ha venido caracterizando, orientado a dar repuesta a tendencias económicas en lugar de dotar aspectos conceptuales, la toma de decisiones y promover avances disciplinares y desarrollo social. 
Urrego, citado por Gómez (2012) menciona:

La educación como función social atiende a la formación de sujetos que aporten al desarrollo social, por lo tanto, no sólo la educación universitaria tiene el compromiso de formar personas, sino que todo el sistema educativo debe aportar a la construcción de sujetos sociales (p. 95).

Siguiendo con la transición hacia la internacionalización, que experimenta la profesión contable, en Colombia, se observa el proceso formativo en NIIF carente de claridad, disperso, acrítico y sin un horizonte definido que dé muestras de la verdadera aplicabilidad en contexto, conducente a establecer patrones o lineamientos que promuevan la reflexión objetiva sobre su adopción (Sierra \& Católico, 2012). Por lo cual se hace necesario reflexionar, si es apropiado el marco metodológico que contiene las estrategias y acciones que definen ¿qué enseñar y cómo enseñar, la norma internacional? para lograr los propósitos de formación requeridos por el actual contexto económico, social y cultural.

En atención a tales consideraciones se presenta este artículo que plantea como pregunta problémica icuál es la pertinencia de las estrategias pedagógicas para la enseñabilidad de las NIIF en el contexto universitario y cuáles son las metodologías empleadas frecuentemente para generar aprendizajes significativos en este tema? En función de responder a tal necesidad, se establece como propósito general e intención central pretendida: analizar la práctica pedagógica empleada por los docentes para insertar la enseñanzaaprendizaje de las NIIF, dentro de los contenidos programáticos en el contexto universitario, de modo que genere aprendizaje relacional significativo y congruente, cómo parte esencial de su formación integral.
Se inserta en el campo de los problemas educativos referidos a la enseñabilidad de la contabilidad, apoyado en el método inductivo que aporta la posibilidad de analizar e interpretar el fenómeno en contexto, toda vez que los aspectos contables constituyen una reflexión en y desde la praxis, por cuanto la realidad de su práctica pedagógica está constituida por hechos observables externos y por símbolos e interpretaciones.

\section{METODOLOGIA}

El abordaje metodológico del presente artículo se basó en el paradigma cuali-cuantitativo, empírico analítico con un diseño de investigación no experimental y corte bibliográfico, lo que en el contexto de la educación superior, posibilitó reconocer y analizar los variados y dinámicos contextos en que se desarrolla el proceso de enseñanza-aprendizaje de la NIIF, a partir de los cuales, se logró definir elementos y características relevantes para un aprendizaje significativo en el estudiante, que bajo la mirada crítica de la acción educativa posibilite la revisión de sus prácticas pedagógicas para adaptarlas de modo que dé respuesta a las necesidades de la sociedad globalizada.

Como instrumento de recolección de información se empleó un cuestionario auto administrado con un diseño cuali-cuantitativo que contiene campos específicos de la investigación favoreciendo la combinación de ambas metodologías para mayor comprensión de la realidad y de acuerdo a las necesidades del estudio, aplicado a docentes y estudiantes de programa de contaduría pública seleccionados intencionalmente, así mismo, observación de prácticas, situaciones y fenómenos, tal como se presentan en el momento de recolección de la información, lo cual permitió su descripción e interpretación. 


\section{DISCUSIÓN}

La intervención social que ejerce la contabilidad dentro del nuevo contexto global demanda dinamismo de los procesos formativos de sus profesionales, de modo que pueda responder exitosamente a las cambiantes y exigentes condiciones sociales retos que suponen una mayor preparación y el desarrollo de competencias integrales, que posibilite hacer uso crítico del conocimiento y un desempeño profesional con sentido social. (Paredes \& Inciarte, 2013)

Cada competencia viene a ser un aprendizaje complejo que integra conocimientos, habilidades, aptitudes, valores y actitudes (Inciarte \& González, 2009). Sin embargo, los docentes y los que están trabajando en sociedades y grupos de empresas directa o indirectamente, porque auditan, son los que están mucho más familiarizados con dichas normas. (Mongue, 2005)

De ésta situación se resalta el proceso educativo instruccional, donde el docente ha pasado de ser un mediador de saberes a ser un aplicador y transmisor de haceres, a través de técnicas e instrumentos que se agotan en el mismo proceso de aplicación. Las modificaciones pedagógicas, son prácticas exóticas, que no están precedidas por la búsqueda de alternativas para la solución de los múltiples problemas que afectan su quehacer universitario (Martínez, 2008). En este sentido algunos autores como Cardona \& Zapata (2005), consideran que en el campo de la educación contable se evidencia un atraso en relación con las demandas del contexto, ello amparado en gran parte por la autonomía universitaria que consagra la ley 30 de 1992, que posibilita planes de estudio contenidos y metodologías integradas sin revisión crítica en contexto.
En línea con lo mencionado Zgaib (2008), considera que:

Los problemas en el proceso de enseñanza-aprendizaje y en el ejercicio profesional empiezan cuando aquellos modelos, en tanto simplificaciones de la realidad, adquieren vida propia, se despojan por completo de los sucesos económicos que debieran servirle de sustento y se vuelven contra sus propios creadores (p.2)

Ante lo expresado, autores como Arroyo (2011), plantean superar el difuso panorama de la enseñabilidad de las NIIF, pasando de un "esquema de elaboración de estados financieros a un enfoque de paradigmas", donde el juicio profesional prima sobre los registros de cargos y abonos, cuyo reconocimiento, medición y revelación, requieren un manejo global de los estándares, en consonancia con la naturaleza de la contabilidad que como ciencia económica requiere el fundamento, la práctica y el conocimiento de las características singulares de los fenómenos financieros.

Para lograr este acometido, se requiere una aproximación del estudiante a un campo específico de conocimiento, para lo cual el docente debe tener un dominio de pensamientos teóricos, prácticos, profundizados con la enseñanza y aprendizaje, que inciden en el proceso formativo. En el caso específico de las NIIF, se observa que sus intenciones formativas han sido conducidas bajo la orientación anglosajona diferente a las colombianas, razón por la que aún se vislumbra confusa su aplicación de acuerdo a las realidades del país, reflejándose en contradicciones en los procesos de enseñanza que requiere una reflexión más profunda para identificar el deber ser en la aplicación contextualizada y los impactos de esta. 
De lo expresado se infiere que la "aprendibilidad, enseñabilidad, y educabilidad" que le otorgan sentido al proceso formativo del contador público, requieren de un acto de comunicabilidad dialógica entre sus actores y los escenarios en los que se desarrolla el aprendizaje, ello requiere de mediaciones pedagógicas y didáctica para conducir los aprendizajes significativos en el estudiante, directamente relacionados con la mirada inter y transdiciplinar que el docente tenga sobre una realidad objeto de enseñanza, que lleva inmersa un sentido cultural, político, económico y social alineado con el proyecto institucional del que hace parte y cuyas competencias promueve. (Fourez, 1997)

En armonía con lo expresado, Correa (2013), explica que el proceso de enseñabilidad ha de ser "dialéctico sistémico" con la realidad para integrar lo general y lo especifico, de modo que la comprensión de lo universal no genere pérdida de identidad propia, lo esperado al conducir procesos de enseñabilidad de las NIIF, es que el docente integre el conocimiento que desea trasmitir, significativo a la realidad práctica del estudiante posibilitando su transformación y promoviendo su intervención para generar cambios sociales. (Gaitán, 2002)

Es de resaltar, que dentro de los elementos de la enseñabilidad, se destaca la no utilización de códigos elaborados, por lo que los procesos aplicados a las NIIF han desvitalizado su finalidad por lo inadecuado de los métodos, técnicas, didácticas y metodologías empleadas para conducir los aprendizajes siendo influenciados por la formación académica, criterio, lenguaje y visión de quien conduce el proceso. (Flórez, 1999)
Opuesto a ello en los escenarios actuales, se observa una desarticulación entre enseñabilidad y aprendibilidad frente a la educabilidad, donde las dos primeras se hayan visiblemente articuladas, pero no ocurre lo mismo con la tercera. La educabilidad, sin embargo, es la razón de ser del proceso formativo, no resulta factible que sean consideradas y analizadas de manera individual, sin considerar las particularidades de sus individuos, sus teorías e interpretaciones de la realidad en contexto, reflexionando críticamente para producir construcciones de nuevos significados. (Gallego \& Pérez, 2011)

En línea con lo mencionado, la enseñanza, la didáctica y la pedagogía son factores de suma importancia que influyen en los procesos de enseñabilidad de las NIIF; ello parte del conocimiento profundo de sus preceptos y el reconocimiento de la realidad contextualizada, considerando las representaciones y experiencias tanto de los docentes como de los estudiantes, lo que trae consigo objetivos específicos para la evaluación y la enseñanza por competencias, aspecto en el que los docentes contables aun requieren de formación. Por lo tanto, para el caso específico de las NIIF, es indispensable conocer cómo se forma y perfecciona al hombre, cómo se puede contribuir al desarrollo de capacidades, habilidades, hábitos, sentimientos y valores a través de la educación, con el fin de incidir positivamente en la formación integral del individuo. (Arbeláez, Correa \& Encarnación, 2012)

Tras lo anteriormente expresado, se hace necesario que los procesos pedagógicos, consideren las diferencias en los modos de aprender e intereses de los estudiantes y considerar las individualidades de cada uno, la cual a pesar de emerger de las interacciones colectivas 
se construye de modo particular, ello favorece relacionarlas con sus estilos de enseñanza. Así, la experiencia educativa se vuelve pertinente, significativa y satisfactoria para todos los que participan en el proceso de enseñanza y aprendizaje. Keefe (citado por Cruz, 2001)

En el proceso de formación para las NIIF, el docente contará con el apoyo institucional, favoreciendo la visibilización de debilidades y la generación de oportunidades de mejora que brinden seguridad a los actores. (Rodríguez, 2008)

La formación integral del contador público, se enfrenta a imponentes desafíos, donde la sociedad contemporánea de profesionales, evidencia una profunda crisis de valores, donde además de trascender en consideraciones económicas, sus actuaciones se ven permeadas de dimensiones de moralidad, espiritualidad y participación ciudadana. (Conferencia mundial de la Organización de las Naciones Unidas para la Educación, la Ciencia y la Cultura - UNESCO, 1998)

\section{RESULTADOS}

Saberes - Conocimiento conceptual $y$ práctico. Los resultados de este ítem evidenciaron un alto reconocimiento por parte de los docentes frente a la importancia de comprender la conceptualización de las NIIF, para orientar procesos de enseñanza aprendizaje con identificación local, en donde se requiere que el docente tenga alto dominio del tema. En este sentido, llama la atención que en el proceso de enseñabilidad de las NIIF, el resultado para ambos casos (docentes y estudiantes, valorados con un $83 \%$, y $51,7 \%$ respectivamente), mostró una tendencia con un mayor nivel de impor- tancia desde los marcos conceptuales y en menor escala las prácticas de campo asociadas $47,4 \%$ y $30,7 \%$ respectivamente), ello podría sugerir alineación con las teorías del empiro-positivismo que tienden a separar lo conceptual de lo metodológico, aislando la teoría de la práctica sin generar transformaciones.

Técnicas pedagógicas en la enseñanza de las NIIF. En este ítem, los resultados evidenciaron que de acuerdo a la percepción de los docentes, se observa mayor tendencia al empleo de técnicas pedagógicas como las lecturas críticas $(57,9 \%)$, talleres $(52,9 \%)$ y clases magistrales $(47,1 \%)$, mientras que los estudiantes observaron con mayor aplicación los talleres y laboratorios $(48,6 \%)$, las exposiciones $(45,9 \%)$ y la mesa redonda (40,5\%). Dado que los estudiantes no son iguales en sus capacidades o en sus intereses cognitivos, el docente ha de considerar en el proceso educativo las individualidades de sus educandos, donde a través del uso de metodologías variadas coadyuve al desarrollo del estudiante como hombre social individualmente considerado, sin pretender ser homogenizado, promoviendo la liberación de prejuicios y generando un pensamiento renovador divergente. (Keefe, citado por Cruz, 2001)

En función de ese propósito, se plantea, que para alcanzar un desempeño idóneo, el docente debe estar en capacidad de estimular al estudiante para que alcance las competencias de saber conocer, saber hacer y saber ser; para ello debe articularse en un plan de trabajo apoyado en estrategia de acuerdo con determinado método, técnicas y actividades de enseñanza-aprendizaje. En la figura 1. Se presentan las relaciones entre métodos, estrategias, técnicas y actividades. 

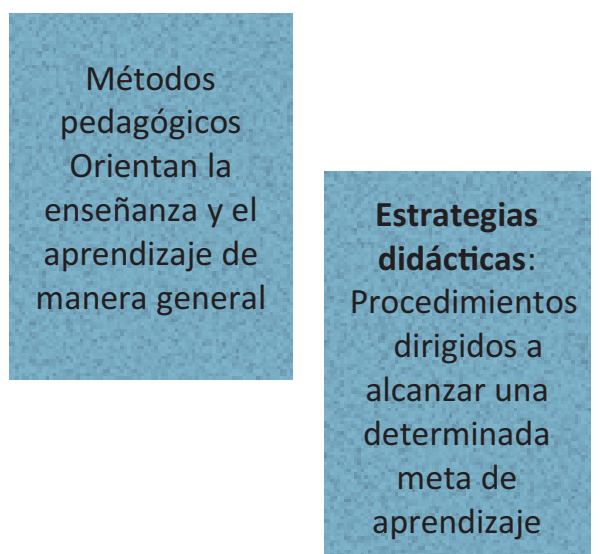

De lo anterior se define, que la aplicación de una pedagogía, didáctica creativa, conducida a través de metodologías activas, promueven aprendizaje significativos para crear actitudes flexibles y generar aportes personales (Seltzer, 2011), método análogo propuesto por Carrasco et al. (2009), basado en proyectos y en actividades.

\section{Competencias a desarrollar en los estudiantes}

Del análisis anterior, se resaltó la baja valoración del componente humanístico dado por ambos actores al proceso de enseñabilidad de las NIIF (docentes $44 \%$ y estudiantes 51,4\%). Ello contrapone el deber de todo proceso educativo de formar a los individuos con orientación humana y social, formar al ciudadano integral y apto para vivir en comunidad, al servicio de la cual

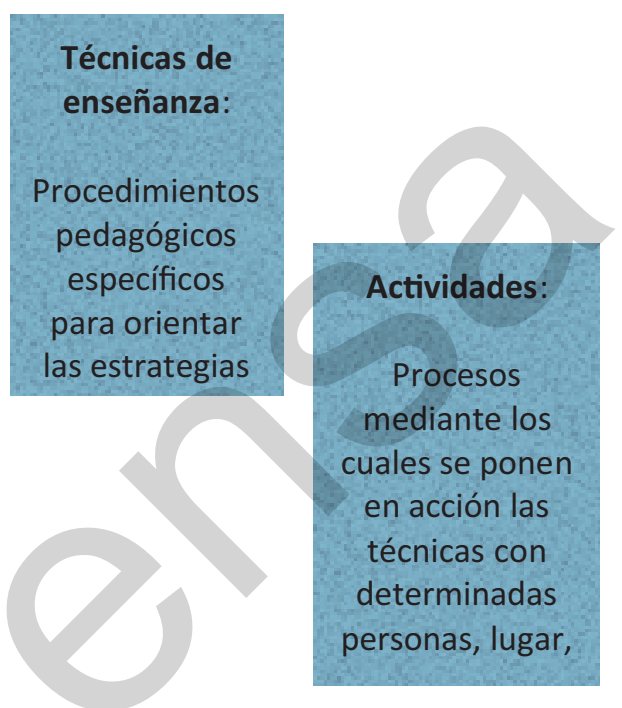

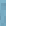

Figura 1. Relación ente métodos, estrategias, técnicas y actividades debe poner sus competencias y saberes; por ende, todo aprendizaje que no se oriente en esta dirección desestima su importancia (Uribe 2004). Por consiguiente, todo proceso de enseñabilidad parte de un acto de comunicación con el entorno en busca de satisfacer las necesidades sociales, de producción y culturales lo cual, nace mediante la relación dialógica con el educando que posibilita el conocimiento como síntesis de discursos conceptuales generados de un aprendizaje significativo una vez hayan sido decodificados los fenómenos, convirtiéndose en soluciones.

\section{Tópicos centrales en la enseñanza de las NIIF}

En los resultados de este ítem, se resaltó una mayor concentración en los referentes teóricos con una valoración del 88,2\%, 
seguido por el marco conceptual 78,9\%, e interpretaciones $77,8 \%$, y en el consolidado general se evidencia la tendencia al $100 \%$ en los niveles alto y superior, con variaciones en los niveles restantes. En correspondencia con ello, se observa alineado a la concepción que el conocimiento, que el marco conceptual no es suficiente para la comprensión de las NIIF, el proceso debe partir de la comprensión de lo particular hacia lo general, conocer a fondo lo que actualmente tiene aplicación en el país para luego pasar a los estándares internacionales.

Lo anterior implica partir desde los principios de contabilidad generalmente aceptados, pasar por el marco conceptual, luego a criterios de reconocimiento y valoración, seguir por comprender la revelación bajo norma local y pasar a norma internacional, para finalmente llegar hasta la elaboración y presentación de estados financieros individuales, consolidados en doble moneda y bajo NIIF, No obstante, no se observó congruencia con estos mismos niveles de importancia las valoraciones dadas sobre los aspectos esenciales de las NIIF que requiere el docente conocer para conducir su enseñanza.

\section{Preferencia de asignatura \\ para la enseñanza de las NIIF}

Se plantea a los estudiantes la posibilidad de tomar una asignatura específica para la enseñanza de las NIIF con un $36,8 \%$ en el nivel alto, mientras que la alternativa de incorporar las NIIF en cada una de las asignaturas del plan de estudios presenta un nivel superior $(78,9 \%)$, este último coincide con el porcentaje arrojado por los docentes quienes manifestaron debe manejarse transversal en todas las asignaturas de formación del contador público; más aún si se considera que como garante de fe pública, deba tener un alto nivel de conocimientos habilidades y valores, dado los nuevos escenarios "demanda un profesional versado en prácticas y estrategias de negocios internacionales" (Cardona \& Zapata, 2005, p. 97). Donde la información contable es el lenguaje universal de los negocios (Adrados, 2007), y los informes financieros son los medios para la toma de decisiones en los mercados. (Gutiérrez \& Núñez, 1996)

\section{Mecanismos de evaluación en la enseñanza de las NIIF}

Los resultados de este ítem evidenciaron mayor relevancia a la solución de ejercicios con un $68,4 \%$, por parte de los docentes y los estudiantes dieron mayor valoración a la evaluación de fundamentos teóricos $48,6 \%$ ponderación que se aleja de las valoraciones anteriores en cuanto a conceptualización docente para conducir procesos de enseñanza aprendizaje.

En ese sentido se menciona, que dados los procesos de enseñanza aprendizaje no deben desconocer la realidad del contexto, una evaluación que mida la formación integral debe incorporar teoría y práctica, de modo que posibilite conectar el mundo económico-financiero con la disciplina contable desde la simulación de problemas reales, Cardona \& Zapata (2005) y Tobón (2007). Por lo cual conjugar, fundamentos teóricos, participación analítica, estudio de casos en la solución de ejercicios es lo deseable.

\section{Nivel de Conocimiento y Apropiación de las NIIF}

Este ítem evidencia una valoración por parte de los docentes del 52,6\% y los estudiantes del 40,5\% como apropiación de las NIIF, como resultado del proceso de enseñanza aprendizaje relación a ello, se establece debilidades para construir aprendizajes significativos, lo cual, implica dificultad 
para trascender las estructuras mentales y prácticas que modifique sus aproximaciones teóricas atribuyéndole sentidos diferentes, lo cual pierde sentido si no contribuye al perfeccionamiento del proyecto ético de vida del estudiante. (Gallego \& Pérez, 2011)

\section{Nivel de aplicación de las NIIF en el ejercicio profesional}

En la práctica profesional se observa una aplicación de NIIF con niveles de orden bajo y medio (docentes $47,4 \%$ y estudiantes $64,9 \%$ ) en el cual se observa dificultad en el estudiante para trascender el conocimiento más allá de su propia realidad, teoría y práctica, por ello este se descontextualiza con el avance y la reflexión crítica sobre su aplicación, por ende sería importante para la continuidad del conocimiento, que el estudiante busque formas de aprender, desaprender y reaprender (Delors, 1996), convirtiéndose gradualmente en un investigador activo de su quehacer, indispensable en el desarrollo de su persona social.

\section{Niveles de empleo de Técnicas pedagógicas}

Los estudiantes consideran que el 40,5\% de los docentes emplean técnicas pedagógicas para conducir los aprendizajes en NIIF mientras el 52\%,6 de los docentes menciona su aplicación. En tal sentido y alineado a ello, se menciona que un proceso de enseñabilidad basado en las lógicas disciplinares sin el apoyo de una pedagogía y una didáctica que se oriente al desarrollo de competencias pertinentes, es académicamente insostenible y con fuerte inclinación al fracaso. Pérez \& Gallego (2011)

\section{Nivel de capacitación docente en las NIIF}

En relación con el nivel de capacitación de los docentes en las NIIF que perciben los estudiantes se evidencia una tendencia de nivel medio (37,8\%), mientras los docentes afirman sentirse capacitados para conducir la enseñanza de las NIIF en un 50\%. El resultado anterior evidenció que el solo dominio conceptual y metodológico de un saber no basta para enseñarlo, por consiguiente el conocimiento que el docente tiene de las NIIF deberá ir acompañado de un saber hacer analizado en contexto, conducido con el apoyo de saberes pedagógicos y didácticos para hacerlo realmente significativo.

Es así como en la dialéctica de la complejidad del proceso de enseñanza aprendizaje de las NIIF, reclama un pensamiento trascendente que problematice constantemente su entorno para generar evolución de conocimiento, obteniendo como resultado el conocimiento científico, tecnológico y la innovación, propósito que sobrepone la pedagogía del sentido común. Por consiguiente se estima que el científico puede ejercer la docencia, pero un elevado número de docentes no pueden actuar como científicos. (Uribe, 2004)

\section{Nivel de calidad de los procesos de actualización docente en las NIIF}

Los estudiantes, consideraron que el nivel de calidad de los procesos de actualización docente en las NIIF es principalmente alto $(43,2 \%)$ y los docentes, consideran con un $84,2 \%$ de calidad, sin embargo se resalta que el $43 \%$ de los encuestados consideró que se encuentran descuidados los seminarios de actualización para egresados, temas donde se requiere mayor atención por parte de los programas contables por cuanto representa la población que visibiliza el impacto en la pertinencia del perfil profesional del contador público.

Al respecto, Mantilla (2011), menciona: 
El desconocimiento de las NIIF hace que los estudiantes no asesoren a empresas del sector catalogadas como Pequeña y Mediana Empresa (PYME) en temas de convergencia contable; que los egresados no tengan las competencias de implementar las NIIF-PYMES o de pertenecer a equipos de trabajo para pertenecer a equipos de trabajo para la implementación de NIIF -PLENAS (p.103).

A manera de síntesis se establece, que para alcanzar los propósitos de formación en el tema de las NIIF, se hace necesario revolucionar la docencia y sus modelos de enseñabilidad de modo que un adecuado proceso pedagógico acompañado de un saber en contexto generen el impacto requerido, para ello se promueve la comprensión de los conceptos y su aplicación en escenarios reales.

\section{CONCLUSIONES}

Los procesos de integración económica que atraviesa Colombia, implican la formación de profesionales con nuevos conocimientos y habilidades en diferentes áreas para que puedan intervenir en las diferentes dinámicas globales. Por ende, el proceso formativo de un contador público que pretenda desarrollar competencias integrales de talla mundial, no puede soportarse en metodologías tradicionales, porque el conocimiento orienta las transformaciones de la sociedad, demandando apertura de pensamiento que posibilite la fácil adaptación al ejercicio profesional y a la vida en comunidad.

Lo anterior implica el diseño de un plan de asignatura profundamente pensado que involucre elementos pedagógicos, axiológicos, culturales, cognitivos metodológicos y didácticos donde toma relevancia el pensamiento crítico en contexto, que involucra dominio de marcos conceptuales, aspectos legales, normas técnicas, uso de tecnologías de información e incluso el manejo de una segunda lengua, de modo que se genere la competencia específica a desarrollar de acuerdo al perfil profesional y los propósitos de cada institución educativa. Por ello, los procesos pedagógicos deben considerar las diferencias en los modos de aprender e intereses de los estudiantes y considerar las individualidades de cada uno, la cual a pesar de emerger de las interacciones colectivas se construye de modo particular.

Se establece que de acuerdo al consenso general se armoniza en afirmar que son más efectivos los procesos de enseñabilidad de las NIIF basados en principios que en reglas, donde se debe partir de la comprensión de los marcos conceptuales y su análisis aplicado en contexto, ello por considerar que dado el menor detalle, posibilitan lugar para el juicio profesional.

Infortunadamente, para su comprensión aún está por definir un marco conceptual fuerte y armónico de aplicación mundial (Casinelli, 2008), ello conlleva a participar en la estandarización, pero entenderla como un proceso mínimo de armonización de la práctica contable, porque el verdadero sentido a esta transición debe ser dirigido a darle bases científicas a la práctica profesional. Se deja abierta la invitación a repensar las temáticas internacionales y su injerencia en el perfil profesional del contador colombiano.

La enseñanza de la contabilidad debe concentrarse más en el saber que en el hacer, por consiguiente, en la tabla 1 . se presenta una serie de recomendaciones con el propósito de trazar un camino o propuesta de solución para la problemática de lograr procesos de enseñabilidad de las Normas Internacionales de Información Financiera (NIIF) de modo que promueva aprendizajes significativos, conforme a las competencias que requiere el contexto y alienados con los perfiles de formación. 
Tabla 1.

Propuesta de enseñabilidad de las NIIF para promover aprendizajes significativos

Diseñar programa de formación para formadores en NIIF para dotar de bases científicas la práctica profesional docente

NIIF basadas en principios y no en reglas lineales y normativas que den lugar al juicio profesional más que al registro estandarizado.

Modelo curricular para la enseñanza de NIIF centrada en ciclos de operaciones y no por cuentas
Sensibilizar al estudiante en su compromiso con la autoformación y aprendizaje continuo, como un principio de responsabilidad social

Considerar modos de aprender e intereses de los estudiantes en el diseño de estrategias pedagógicas

Alineación de las competencias y estrategias pedagógicas al modelo pedagógico y los propósitos de formación

Adaptación del plan de asignatura profundamente pensado que involucre elementos pedagógicos, axiológicos, culturales, cognitivos metodológicos y didácticos

Evaluación que incluya en el aprendizaje de las NIIF, competencias genéricas necesarias para el adecuado desempeño profesional que favorezca la construcción dialógica y escrita de argumentos de valor.

Aaplicación de alternativas de enseñanza aprendizaje a método hermenéutico-dialéctico

Involucrar a los docentes del programa en la revisión y proyectos de mejoramiento de los resultados de las pruebas Saber Pro

Involucrar didáctica que requieran soporte epistemológico, revisión de postulados, teorías, principios de contabilidad y económicos

Transmitir reflexiones abiertas que produzca información objetivamente neutra sin sesgo de interpretación personal que favorezca el análisis y construcción de juicios de valor individuales

Implementar conversatorios, jornadas de discusión y talleres docentes para compartir experiencias de casos en la práctica pedagógica de modo que pueda construirse un marco conceptual armónico y solido

Problematizar constantemente la realidad para analizarla de manera crítica y reflexiva, con juicio de comparación e identificación de particularidades

Sensibilizar sobre el verdadero propósito de la estandarización, entendida como un proceso mínimo de armonización de la práctica contable

Incorporar en el proceso formativo últimas tendencias en materia de investigación académica en temas internacionales y de NIIF así como los desarrollos de los organismos profesionales, discusión de pronunciamientos disciplinarios

Discusión de lecturas y videos y análisis de situaciones reales (dilemas éticos)

Laboratorios de simulación y ambientes de trabajo en entorno internacional que estimulen implementación de NIIF en diferentes fases

Conversatorios con docentes invitados de otros países tanto académicos como profesionales en ejercicio.

Debates abiertos entre actores (gremios, organismos de control, juntas, académicos y estudiantes).

Elaborar material didáctico docente para estudio, tales como cartillas y guías, que facilite la comprensión en temas específicos y suministre bibliografía de apoyo para posteriores lecturas

Seminarios y pláticas profesionales con invitados sobre estos temas donde se de participación activa de estudiantes y propuestas.

Didácticas que impliquen uso intensivo de tecnología y manejo de una segunda lengua

Diseñar programa de extensión en NIIF a la comunidad a través de la creación de laboratorios contables como una ventana de observación de prácticas para los estudiantes de pregrado y como un servicio social.

Monitoreo al proceso formativo de las NIIF para controlar avances y corregir retrocesos.

Equilibrar la teoría y la práctica de modo que posibilite la superación de la falsa antinomia en los procesos de enseñanza aprendizaje de las NIIF muy teóricos o muy prácticos.
Privilegiar la formación humanista de la persona en su proyección profesional internacional sin pérdida de identidad local 


\section{REFERENCIAS}

Adrados, M. (1997). Sistemas de información contable. Ensayos sobre contabilidad y economía. Contabilidad de gestión y economía financiera. Madrid: instituto de contabilidad auditoría de cuentas. Ministerio de economía y Hacienda.

Arbeláez, D., Correa, L. \& Encarnación, A. (2012). Problemas, retos, competencias y aspectos de calidad en la enseñanza de la auditoría: una aproximación a las percepciones de estudiantes y docentes. Revista Latinoamericana de Estudios Educativos, 42(1), 25-54.

Arroyo, A. (2011). Normas Internacionales de Información Financiera y el Peritaje Contable. Contabilidad y Negocios. Recuperado de: http://www.redalyc.org/articulo. oa?id=281622822006

Avellaneda, A. (2010). Factores de incidencia de la Ley 1314 de 2009 en la educación contable Colombiana. Criterio Libre, 8(12), 209-237.

Cardona, J., Zapata, M. (2005). Educación Contable Antecedente, actualidad y prospectiva. Universidad de Antioquia, Medellín.

Carrasco, F. \& Larrinaga, C. (1996). El poder constitutivo de la contabilidad: consideraciones sobre la cuestión medio ambiental. Ensayos sobre contabilidad y economía. 2, 65-84.

Carrasco, A., Donoso, J., Duarte, T., Hernández, J., López, R. \& Núñez, C. (2009). Aprendizaje basado en proyectos versus aprendizaje basado en actividades: una experiencia en la elaboración y análisis de los estados financieros. VI Jornadas de
Docencia, Asociación Española de Profesores Universitarios de Contabilidad, ASEPUC. Recuperado de: http://www.asepuc.org/banco/11.pdf

Casinelli, H. (2008). NIIF/IFRS, Normas internacionales de información financiera. La globalización del lenguaje de los negocios. Buenos Aires: Aplicación tributaria S.A.

Castillo, S. \& Cabrerizo, J. (2010). Evaluación educativa de aprendizajes $y$ competencias. Madrid: Pearson Educación

Correa, C. (2013). Currículo Transdisciplinar y Práctica Pedagógica Compleja. Barranquilla, Colombia: Ediciones Universidad Simón Bolívar.

Cruz, D. (2001). Enseñanza y aprendizaje en la educación superior: un reto para el siglo XXI, Puerto Rico: Universidad de Puerto Rico.

Delors, J. (1996). La educación encierra un tesoro. UNESCO. Recuperado de: http://www.unesco.org/education/pdf/DELORS_S.PDF

Fourez, G. (1997) "Alfabetizacion cientifica y tecnologica acerca de la finalidad de la enseñanza de las ciencias" Buenos aires: Ediciones Colihue S.R.L.

Flórez, R. (1999). "Enseñabilidad y pedagogía”. (Táchira). Acción Pedagógica. 08(01), 34-37.

Freire, P. (1970). Pedagogía del Oprimido. Montevideo, Tierra Nueva, Buenos Aires: Editorial Siglo XXI Argentina Editores

Gaitán, C. (2002). "La enseñabilidad de las ciencias sociales. En: Memorias sobre actualización y mejoramiento curricular". Bogotá: Beta Impresores, 165-176. 
Gallego, R. \& Pérez, R. (2011) “Aprendibilidad, enseñabilidad y educabilidad: una discusión". Recuperado de: http://aprendeenlinea.udea.edu. co/revistas/index.php/revistaeyp/ article/viewFile/5861/5274

Giroux, H. (1990). Los Profesores como Intelectuales: Hacia una pedagogía crítica del aprendizaje. Barcelona: Ed. Paidos.

Gómez, M. (2005). Breve introducción al estado del arte de la orientación critica en la disciplina Contable. Porik An, 10, 13-37.

Gómez, Y. (2012). Educabilidad: una resignificación conceptual en contabilidad desde la educación crítica. Contaduría Universidad de Antioquia, 61, 91-110.

Gutiérrez, I. \& Núñez, M. (1996). La información externa como elemento de supervivencia empresarial. Madrid: Editorial: Instituto de Contabilidad y Auditoría de Cuentas

Habermas, J. (2002). "Teoría de la acción comunicativa". ( $1^{\mathrm{a}}$. ed.) Méjico: Taurus

Inciarte, N. \& González, L. (2009). Competencias del docente de educación superior como mediador en los procesos de investigación y evaluación de los aprendizajes. Omnia, 15(2), 9-55.

Mantilla, S. (2011), Estándares/Normas Internacionales de información financiera (NIIF). Segunda Edición. Bogotá Colombia: ECO Ediciones.

Martínez, G. (2001). El estatuto de Cientificidad de la contabilidad: un debate inconcluso. Revista Porik $A n, 5$ y $6,41-70$.
Martínez, G. (2008). La educación contable: Encrucijada de una formación monodisciplinaria en un entorno complejo incierto, Popayán, Colombia: Editorial Unicauca

Mattessich, R. (2003). Contabilidad: ¿cisma o síntesis? El desafío de la teoría condicional normativa. Revista partida doble, 144, 104-119.

Monge, P. (2005). Las Normas Internacionales de Contabilidad Actualidad Contable. Actualidad Contable FACES, 8(10), 35-52.

Paredes, I, \& Inciarte, A. (2013). Enfoque por competencias. Hacia la integralidad y el desempeño profesional con sentido social y crítico. Omnia, 19(2), 125-138.

Rodríguez, J. (2008). Analistas simbólicos, profesionales del futuro, Revista de la Facultad de Ciencias Contables Económicas y Administrativas, 7.

Seltzer, J. (2011). La aplicación de una didáctica creativa en la enseñanza de la contabilidad. Revista Fuentes, 3, 87106.

Sierra, Z, \& Católico, D. (2012), Estandarización contable en Colombia: avances y retos en la formación profesional contable. Recuperado de: http://contaduria. usta.edu.co/images/documentos/activos/12-1.pdf

Tobón, S. (2007). Formación Basada en Competencias. Bogotá: ECOE Ediciones Ltda.

Organización de las Naciones Unidas para la Educación, la Ciencia y la Cultura UNESCO, (1998). Conferencia Mundial sobre la Educación Superior. La educación superior en el siglo XXI: visión y acción. París, tomo I, Informe final, 5-9 de octubre. Recuperado de: $h t t p: / / w w w$. unesco.org/education/educprog/wche/ declaration_spa.htm 


$$
\left\langle e^{e^{e^{g^{2}}}}\right.
$$

